\title{
Site-specific specimen preparation technique for atom probe analysis of grain boundaries
}

\author{
D. Lawrence*, K. Thompson*, D. J. Larson* \\ * Imago Scientific Instruments Corp. 6300 Enterprise Ln, Suite 100, Madison, WI 53719
}

The 3-D atom-probe provides unparalleled compositional analysis of grain boundaries within polycrystalline materials. The adaptation of the local electrode and wide field detector schemes has increased the attainable field of view to as big as $200 \mathrm{~nm}$ in diameter for certain materials [1]. However, many materials microstructures are composed of grains whose size is so large (tens of microns) that even such a wide field of view analysis may be insufficient to guarantee examination of a uniquely chosen grain boundary. Recently developed special methods employing a focused ion beam (FIB) system [2,3], may now be employed to capture the desired region(s) of interest within the specimen tip in a single sample preparation iteration.

This rapid, site-specific sample preparation was performed with a standard tripod polisher, polishing wheel, diamond lapping film, and a FEI 620 dual beam FIB. Samples were subsequently analyzed in the LEAP3000 atom probe with an $80 \mathrm{~mm}$ flight path and $40 \mathrm{~mm}$ detector.

The first step is to transform the bulk sample into a $0.5 \mathrm{~mm} \times .05 \mathrm{~mm} \times 10 \mathrm{~mm}$ lamella. The sample is mechanically reduced into a wedge shape using routine TEM tripod polishing; stepping through diamond lapping film grain sizes (30um, 15um, 6um, 3um, 1um). The finished wedge apex is on the order of 10 microns thick. The sample is then crimped into a $10 \mathrm{~mm}$ length of copper tubing with approximately $5 \mathrm{~mm}$ of sample exposed. The sample and stub are then placed into an electropolishing rig and thinned to 3-5um. This further polishes the sample, removing mechanical artifacts introduced during the mechanical polishing, and controlling the final pre-FIB thickness, see Fig. 1.

A wet metallographic etch is employed to reveal grain boundaries, Fig. 2, and the specific grain boundary for analysis is identified. Material on either side of the grain boundary is removed via milling at high (13 nA) beam currents in the FIB. This forms a tip region that is appropriate for atom-probe analysis, Figs. 3 and 4 . Atom probe analysis with a local electrode typically requires $\sim 50$ microns of clearance in all directions from the tip. For analysis in a system that does not utilize a local electrode, all of the excess material must be removed. Choosing a grain structure near the vertical edge of the sample is preferable since less bulk material need be removed. Once the bulk material is removed from the tip region, annular milling at lower beam currents (350 pA) shapes a high-quality atom-probe tip around the grain boundary feature of interest $[2,3]$. Because the local electrode localizes the field evaporation phenomena [1], multiple tips can easily be produced from a sample with the above listed dimensions, Figs. 3 and 4. The sample is now suitable for atom-probe analysis, Fig 5. Results, Fig. 6, confirm a high-quality grain boundary analysis including compositional quantification of elements between the grains.

The authors gratefully acknowledge the help provided by Ray O'neill, Mohammed Ali, Stephanie Kostrna, Rich Martens, Terry Mengelt in accomplishing this work.

[1] T.F. Kelly and T.T. Gribb, et al. Microscopy and Microanalysis 10 (2004) 373

[2] M. K. Miller, K. F. Russell and G. B. Thompson, Ultramicroscopy, 102 (2005) 287-298.

[3] D.J. Larson, et al, Microscopy and Microanalysis 7, (2001) 24. 

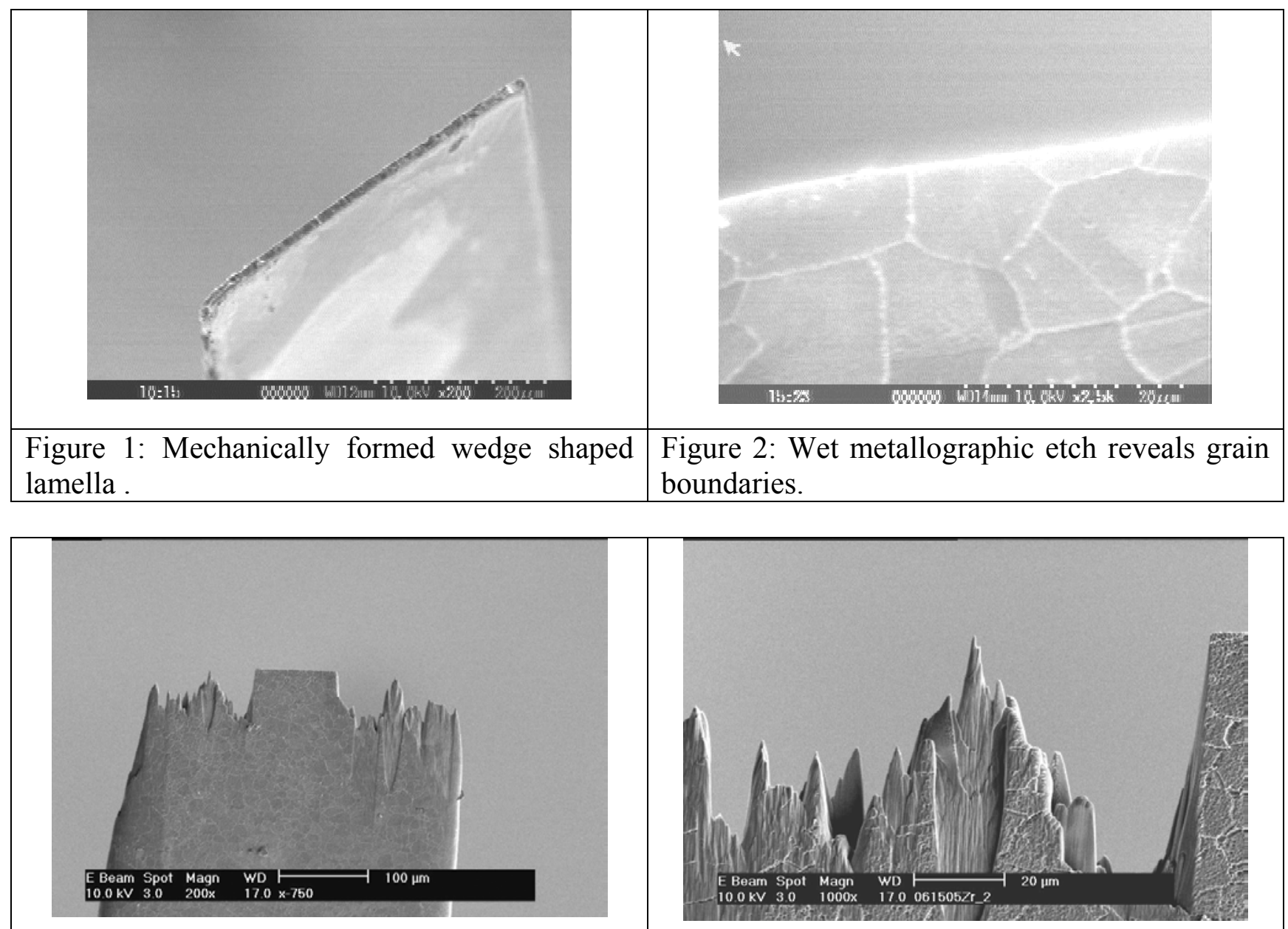

Figure 3: Focused ion beam milling around specifically chosen grain boundaries.

Figure 4: Close up image of tip containing chosen grain boundary.

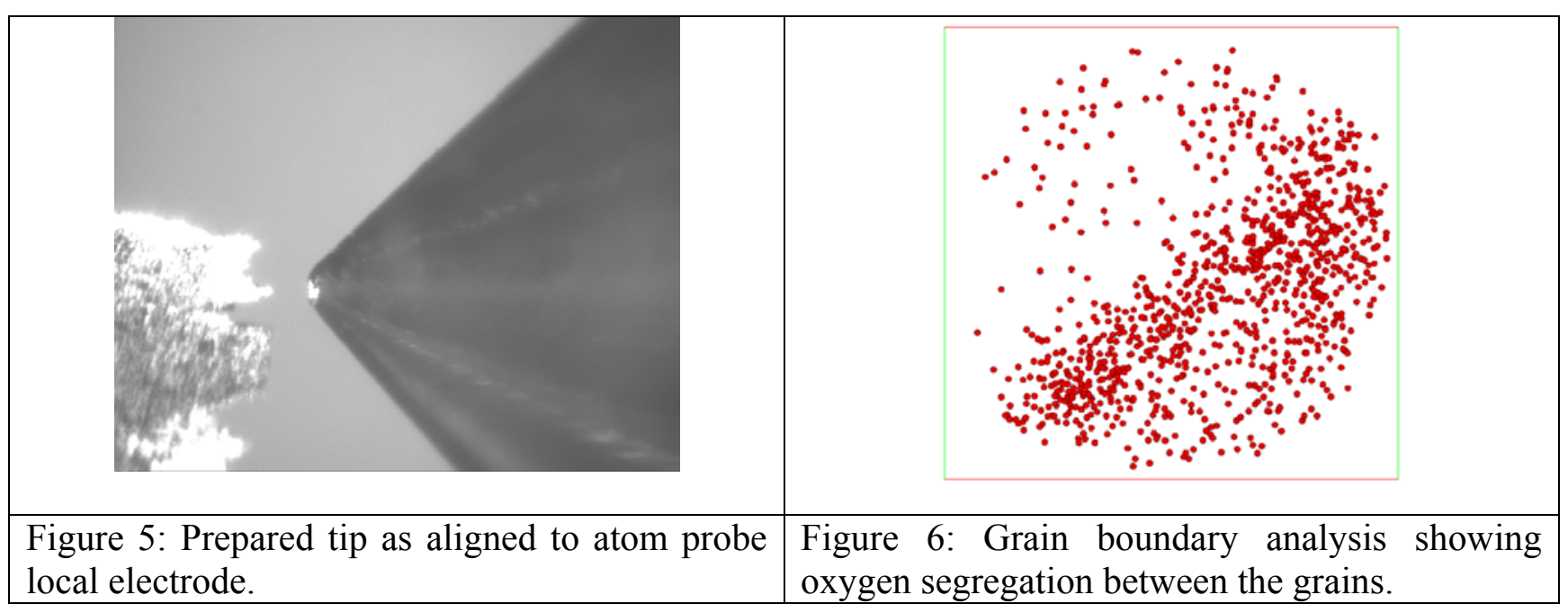

